\title{
Sodium-Glucose Cotransporter-2 Inhibitors and Cardiovascular Disease: Lessons and the Future
}

\author{
Abdulhalim J.A. Kinsara, FRCP, FACC, FESC; Atif Al Qubbany, MD; Wail Alkashkari, MD \\ Department of Cardiology, Ministry of National Guard-Health Affairs, King Saud Bin Abdulaziz University for Health Sciences, COM-WR, King Abdullah International \\ Medical Research Center, Jeddah, Saudi Arabia
}

"Corresponding author

Abdulhalim J.A. Kinsara, FRCP, FACC, FESC

Assistant Professor, Department of Cardiology, Ministry of National Guard-Health Affairs, King Saud Bin Abdulaziz University for Health Sciences, COM-WR, King Abdullah International Medical Research Center, Jeddah, Saudi Arabia; E-mail: akinsara@yahoo.com

\section{Article information}

Received: August 19 th $^{\text {2020; Revised: September } 8^{\text {th }}, 2020 \text {; Accepted: September 14 }}$, 2020; Published: September $18^{\text {th }}, 2020$

\section{Cite this article}

Kinsara AJA,AI Qubbany,Alkashkari W. Sodium-glucose cotransporter-2 inhibitors and cardiovascular disease: Lessons and the future. Intern Med Open J. 2020; 4(I): I0-12. doi: I0.17|40/IMOJ-4-II3

\section{| ABSTRACT |}

Diabetes mellitus (DM), an epidemic non-communicable disease, is associated with macro- and micro-vascular complications which may result in sudden cardiac death at a young age. Sodium-glucose cotransporter-2 inhibitors (SGLT2-I) emerged as a new therapeutic option for managing DM with cardiovascular complications as well as diabetic patients with multiple risk factors. Three drugs in this class significantly reduced cardiovascular mortality and heart failure events, in both type 2 diabetes mellitus and non-diabetic patients with a reduced ejection fraction, to prevent heart failure related hospitalisation. Evidence of kidney protection was another major advantage provided in more than one study. We reviewed recent SGLT2-I related literature and discuss the benefits beyond the cardiac system.

\section{Keywords}

Diabetes mellitus (DM); Cardiovascular disease; Sodium-glucose cotransporter-2 inhibitors (SGLT2-I); Renal protection.

\section{INTRODUCTION |}

$\mathrm{D}$ iabetes mellitus (DM) is a growing problem in developing and developed countries. It is linked to macro-vascular complications such as myocardial infarction, stroke and peripheral artery disease. In addition to micro-vascular complications in the form of retinopathy, nephropathy, neuropathy, or a combination of any of these complications, the inevitable outcome is heart failure (HF). This close association highlighted the need to measure the effect of diabetic medication on the cardiovascular system. For example, biguanides demonstrated a positive effect on the cardiovascular system as well the glucagon-like peptide agonists. Recently three sodium-glucose cotransporter-2 inhibitors (SGLT2-I) had shown a promising outcome.

\section{DISCUSSION}

The pathophysiological cardiac injury due to DM occurs through various mechanisms, with two currently accepted. The first is mediated through atherosclerosis, with DM considered a high-risk for atherosclerotic events resulting in myocardial wall damage. ${ }^{1}$ The outcome is heart failure with a reduced ejection fraction. The second is independent of atherosclerosis and mediated by a direct inflammatory effect in the micro-vascular system and myocardium with subsequent fibrosis, also called diabetic cardiomyopathy. ${ }^{2}$ Both mechanisms result in HF. Myocardial damage leading to left ventricle $(\mathrm{LV})$ dysfunction and $\mathrm{HF}$ is an early and often undetected complication of DM. According to Faden et $\mathrm{al}^{3}$ two thirds of patients who had DM for more than 5-years, exhibited a variable grade of LV dysfunction. ${ }^{3}$ In addition, undiagnosed HF was detected in $28 \%$ of patients diagnosed with diabetes during cardiac screening. ${ }^{4}$

Diabetes mellitus can predispose the patient to heart failure with a preserved ejection fraction (HFpEF) or reduced ejection fraction (HFrEF). In addition to this, the high rate of subclinical heart failure and diastolic dysfunction in diabetic patients contribute to the high rate of heart failure related hospitalization and cardiovascular (CV) death in this population. ${ }^{5,6} \mathrm{~A}$ recent study 
in Denmark highlighted the risks DM patients are exposed to, with an incidence of $9 \%$ of sudden cardiac death in 14,294 deaths over a 10 -year period. It should be noted that the risk of sudden death in the DM group younger than 35-years, was 21.9 per 100,000 person-years compared to 2.6 in the group without DM, and 119.8 versus 19.7 in an older age group (36-49-years). The study emphasized the importance of risk factor control, particularly in the younger DM population.

The Swedish Diabetes Register indicated that the decline in the $\mathrm{CV}$ death rate in the DM population is slowing. ${ }^{8}$ In addition, the decline in major CV related deaths and atherosclerotic events did not correlate with the decline in heart failure or arrhythmia. ${ }^{9}$ The 1-year and 3-year all-cause mortality estimates of the Heart Failure Risk Calculator, listed DM as the most important risk stratifier after age and a low ejection fraction in terms of an adverse prognosis. ${ }^{10}$ Due to these challenges, the guideline highlights the implementation of an aggressive risk reduction strategy in stage A and stage B of HF-I, the development and the progression of the disease, to prevent HF in diabetes.

Due to the seriousness of these concerns, the unique effect noted with sodium-glucose cotransporter-2 (SGLT2) inhibitors, resulted in the initiation of several SGLT2 inhibitor trials (Table 1). Three trials compared a DM population in terms of primary (multiple risk factors) and secondary prevention (established cardiovascular disease (CVD)). The Empagliflozin Cardiovascular Outcome Event Trail in Type 2 Diabetes Mellitus Patients (EMPAREG OUTCOME) recruited patients requiring secondary prevention, and in the CANagliflozin CardioVascular Assessment Study (CANVAS) program and dapagliflozin effect on cardiovascular events - thrombolysis in myocardial infarction 58 (DECLARETIMI 58), 34\%-59\% of the participants were in the primary arm. ${ }^{11}$ ${ }^{13}$ SGLT2 inhibitors decrease glucose and sodium reabsorption in the proximal tubule as well as nephron hyperfilteration, which enhance urinary glucose and sodiumexcretion. ${ }^{14}$ SGLT2-I modulates several factors related to $\mathrm{CV}$ risk, including a decreased level of gl-

\begin{tabular}{lccc}
\hline Table I. Summary of the Important & Differences between the Three SGLT2-I Trials \\
\hline & $\begin{array}{c}\text { EMPA-REG } \\
\text { OUTCOME }\end{array}$ & $\begin{array}{c}\text { CANVAS } \\
\text { Program }^{2}\end{array}$ & $\begin{array}{c}\text { DECLARE- } \\
\text { TIMI 58 }\end{array}$ \\
\hline No. of patients & 7000 & 4330 & 17150 \\
\hline Duration of trial (y) & 5 & $\geq 4$ & 6 \\
\hline Age, years, mean (SD) & $63 \pm 9$ & 63 & $63.9 \pm 6.8$ \\
\hline Micro-/macro-albuminuria (\%) & 40.6 & 30.2 & 30.2 \\
\hline eGFR, mean (mL/min/I.73 $\left.\mathrm{m}^{2}\right)$ & 74.1 & 76.5 & 85.2 \\
\hline \% Primary prevention & 100 & 34 & 59 \\
\hline \% Secondary prevention & 14 & 14 & 41 \\
\hline 3-point MACE risk & 38 & Neutral & Neutral \\
\hline CV death & Neutral & Neutral & Neutral \\
\hline Non-fatal myocardial infarction & Neutral & Neutral & Neutral \\
\hline Non-fatal stroke & 35 & 33 & 27 \\
\hline HF hospitalization & 10 & 100 & 10 \\
\hline Dose & & & \\
\hline & & & 27 \\
\hline
\end{tabular}

ucose and insulin, sympathetic nervous system activity, blood pressure and arterial stiffness, weight and visceral adiposity, oxidative stress, triglyceride, uric acid, albuminuria and increased Highdensity lipoprotein (HDL). The DECLARE-TIMI 58 demonstrated that the benefit of SGLT2-I is functional in the primary and secondary prevention categories. ${ }^{15}$ The notable conclusion was the improvement observed in the hospitalization for heart failure (HHF) in all three studies: the DECLARE, CANVAS and EMPAREG. The SGLT2-I demonstrated a benefit in terms of reducing $\mathrm{CV}$ death and HHF in patients with HFrEF, the effect that was seen early and continued throughout the trial. ${ }^{16}$ In addition, Dapagloflozin for example, resulted in a major adverse cardiovascular events (MACE) relative risk reduction of $16 \%$ and an absolute risk reduction of $2.6 \%$, compared to placebo. Though we need $32 \mathrm{pa}$ tients to prevent one death with ramipril, 56 with simvastatin and 71 with liraglutide, only 39 are required to show a similar effect with empagloflozin.

Intensive treatment with anti-diabetic agents showed some benefit in terms of atherosclerosis endpoints (myocardial infarction/coronary heart disease (MI/CHD)), but no definite improvement in stroke or mortality. ${ }^{17}$ The newer antihyperglycemic agents, however, have shown CV outcome benefits in multiple studies and the CV benefit may be a class effect for SGLT2. ${ }^{18}$ The European Society of Cardiology (ESC) guideline provides a strong recommendation for class 1 , stating that this group should be added to the treatment of patients with DM with CVD or at very high-risk of CVD. ${ }^{19}$ Another class 1 indication for DM patients, was to add SGLT2-I to reduce hospitalization due to heart failure. SGLT2-I is associated with a lower risk in terms of renal end points and recommended if the glomerular filtration rate (GFR) is $30-90 \mathrm{ml} / \mathrm{min} .{ }^{19}$ In contrast to the renal protective effect observed in the renin-angiotensin-aldosterone system (RAAS) blockade, where the effect is mainly on the afferent constriction, SGLT2-I function through efferent vasodilation. SGLT2-I decrease glomerular pressure, reducing albuminuria. ${ }^{20}$ Of interest is that the finding that the Dapagliflozin benefits more than DM patients, as the non-DM group also had a reduced HHF. The benefits surpassed the rare side effects reported, including recurrent genital infection, urinary tract infections and volume depletion. Lower limb amputation, fracture and euglycemic diabetic ketoacidosis, were rarely reported.

\section{CONCLUSION}

In conclusion, SGLT2-I are recommended for the prevention of $\mathrm{HF}$ hospitalisation in patients with DM and established CV disease or with multiple risk factors. A significant reduction in CV mortality and HF events was noted in both DM and non-DM patients with a reduced ejection fraction. Research is ongoing to demonstrate a similar effect in patients with a preserve dejection fraction.

\section{CONFLICTS OF INTEREST}

The authors declare that they have no conflicts of interest. 


\section{REFERENCES}

1. De Simone G, Devereux RB, Chinali M, Lee ET, Galloway JM, Barac A, et al. Diabetes and incident heart failure in hypertensive and normotensive participants of the strong heart study. $J$ Hypertens. 2010; 28(2): 353-360. doi: 10.1097/HJH.0b013e3283331169

2. Redfield MM. Heart failure with preserved ejection fraction. N Engl J Med. 2016; 375(19): 1868-1877. doi: 10.1056/NEJMcp1511175

3. Faden G, Faganello G, De Feo S, Berlinghieri N, Tarantini L, Di Lenarda A, et al. The increasing detection of asymptomatic left ventricular dysfunction in patients with type 2 diabetes mellitus without overt cardiac disease: Data from the SHORTWAVE study. Diabetes Res Clin Pract. 2013; 101(3): 309-316. doi: 10.1016/j. diabres.2013.07.004

4. Boonman-de Winter LJ, Rutten FH, Cramer MJ, Landman MJ, Liem AH, Rutten GE, et al. High prevalence of previously unknown heart failure and left ventricular dysfunction in patients with type 2 diabetes. Diabetologia. 2012; 55(8): 2154-2162. doi: $10.1007 / \mathrm{s} 00125-012-2579-0$

5. McMurray JJ, Gerstein HC, Holman RR, Pfeffer MA. Heart failure: A cardiovascular outcome in diabetes that can no longer be ignored. Lancet Diabetes Endocrinol. 2014; 2(10): 843-851. doi: 10.1016/S2213-8587(14)70031-2

6. Gilbert RE, Krum H. Heart failure in diabetes: Effects of antihyperglycaemic drug therapy. Lancet. 2015; 385(9982): 2107-2117. doi: 10.1016/S0140-6736(14)61402-1

7. Lynge TH, Svane J, Pedersen-Bjergaard U, Gislason G, TorpPedersen C, Banner J, et al. Sudden cardiac death among persons with diabetes aged 1-49 years: A 10-year nationwide study of 14294 deaths in Denmark. Eur Heart J. 2020; 41(28): 2699-2706. doi: 10.1093/eurheartj/ehz891

8. Rawshani A, Rawshani A, Franzén S, Eliasson B, Svensson AM, Miftaraj M, et al. Mortality and cardiovascular disease in type 1 and type 2 diabetes. N Engl J Med. 2017; 376(15): 1407-1418. doi: 10.1056/NEJMoa1608664

9. Cheng YJ, Imperatore G, Geiss LS, Saydah SH, Albright AL, Ali $\mathrm{MK}$, et al. Trends and disparities in cardiovascular mortality among U.S. adults with and without self-reported diabetes, 1988-2015. Diabetes Care. 2018; 41(11): 2306-2315. doi: 10.2337/dc18-0831

10. Pocock SJ, Ariti CA, McMurray JJ, Maggioni A, Køber L, Squire IB, et al. Predicting survival in heart failure: A risk score based on 39372 patients from 30 studies. Eur Heart J. 2013; 34(19): 1404-1413. doi: 10.1093/eurheartj/ehs337
11. Zinman B, Wanner C, Lachin JM, Fitchett D, Bluhmki E, Hantel S, et al. Empagliflozin, cardiovascular outcomes, and mortality in type 2 diabetes. $N$ Eng J Med. 2015; 373(22): 2117-2128. doi: 10.1056/NEJMoa1504720

12. Neal B, Perkovic V, Mahaffey KW, de Zeeuw D, Fulcher G, Erondu N, et al. Canagliflozin and cardiovascular and renal events in type 2 diabetes. $N$ Engl J Med. 2017; 377(7): 644-657. doi: 10.1056/NEJMoa1611925

13. Wiviott SD, Raz I, Bonaca MP, Mosenzon O, Kato ET, Cahn A, et al. Dapagliflozin and cardiovascular outcomes in type 2 diabetes. N Engl J Med. 2019; 380(4): 347-357. doi: 10.1056/NEJMoa1812389

14. Sattar N, McLaren J, Kristensen SL, Preiss D, McMurray JJ. SGLT2 Inhibition and cardiovascular events: Why did EMPAREG outcomes surprise and what were the likely mechanisms? $D i$ abetologia. 2016; 59(7): 1333-1339. doi: 10.1007/s00125-016-3956-x

15. Verma S, Jüni P, Mazer CD. Pump, pipes, and filter: do SGLT2 inhibitors cover it all? Lancet. 2019; 393(10166): 3-5. doi: 10.1016/ S0140-6736(18)32824-1

16. Kato ET, Silverman MG, Mosenzon O, Zelniker TA, Cahn A, Furtado RHM, et al. Effect of dapagliflozin on heart failure and mortality in type 2 diabetes mellitus. Circulation. 2019; 139(22): 2528-2536. doi: 10.1161/CIRCULATIONAHA.119.040130

17. Ray KK, Seshasai SR, Wijesuriya S, Sivakumaran R, Nethercott S, Preiss D, et al. Effect of intensive control of glucose on cardiovascular outcomes and death in patients with diabetes mellitus: A meta-analysis of randomised controlled trials. Lancet. 2009; 373(9677): 1765-1772. doi: 10.1016/S0140-6736 (09) 60697-8

18. Furtado RHM, Bonaca MP, Raz I, Zelniker TA, Mosenzon O, Cahn A, et al. Dapagliflozin and cardiovascular outcomes in patients with type 2 diabetes mellitus and previous myocardial infarction. Circulation. 2019; 139(22): 2516-2527. doi: 10.1161/CIRCULATIONAHA.119.039996

19. Grant PJ, Cosentino F. The 2019 ESC Guidelines on diabetes, pre-diabetes, and cardiovascular diseases developed in collaboration with the EASD: New features and the 'Ten Commandments' of the 2019 Guidelines are discussed by Professor Peter J. Grant and Professor Francesco Cosentino, the Task Force chairmen. Eur Heart J. 2019; 40(39): 3215-3217. doi: 10.1093/eurheartj/ehz687

20. Cherney DZ, Perkins BA, Soleymanlou N, Maione M, Lai $\mathrm{V}$, Lee $\mathrm{A}$, et al. Renal hemodynamic effect of sodium-glucose cotransporter 2 inhibition in patients with type 1 diabetes mellitus. Circulation. 2014; 129(5): 587-597. doi: 10.1161/CIRCULATIONAHA.113.005081 